\title{
Estudio Clínico Piloto: Cuñas de Titanio, una Alternativa para Mejorar la Estabilidad Primaria de Implantes
}

\author{
Titanium Wedges, an Alternative to Improve Implant's \\ Primary Stability. Pilot Clinical Study
}

Valenzuela $C^{1}$, JM Carrascoํ․ Silva $\mathrm{M}^{1}$, Yáñez $\mathrm{P}^{1}$

RESUMEN

Al tallar un lecho óseo, por varias razones, puede quedar una preparación de mayor diámetro al del implante que será insertado, en esta situación no es posible roscarlo en el hueso, ya que este tornillo de titanio no logra estabilidad inicial; cuando esto ocurre, es necesario el uso de cilindros roscados de titanio de mayor diámetro. En caso de no contar con este tornillo de diámetro mayor, podemos colocar en el lecho óseo el implante que queda suelto, y una cuña de titanio puede ser introducida fuertemente entre este y la pared alveolar, logrando de esta manera la estabilidad inicial requerida para conseguir la oseointegración deseada.

Rev. Clin. Periodoncia Implantol. Rehábil. Oral Vol. 3(1); 39-42, 2010.

Palabras clave: Implantes, cuñas, titanio, oseointegración.

\section{ABSTRACT}

When ridge bone perforations, for any reason, result wider than implant diameter, we may not be able to fix the fixture into bone walls. When this happens it becomes necessary to use a larger diameter titanium cylinder. In this article we propose, instead of using a wider titanium cylinder, the use of mini titanium wedges which can be pushed strongly between implant and bone walls, to keep fixture still while oseointegration takes place.

Rev. Clin. Periodoncia Implantol. Rehábil. Oral Vol. 3(1); 39-42, 2010.

Key words: Implants, wedge, oseointegration.

\section{INTRODUCCIÓN}

La implantología moderna se remonta a fines de los años 60 , cuando Brånemark sienta las bases de la oseointegración y describe los primeros implantes de titanio(1). Desde entonces y hasta nuestros días, dicha ciencia ha evolucionado de una manera sorprendente y vertiginosa demostrando ser una forma eficaz y segura para reponer dientes perdidos, la cual se hace cada vez más habitual dentro de la práctica odontológica actual(2).

El proceso que hace posible la realidad de un implante se conoce como oseointegración, y consiste en la formación de una fuerte unión entre el titanio y el hueso. Dicho proceso se define según Brånemark como "una conexión directa estructural y funcional entre el hueso vivo, ordenado, y la superficie de un implante sometido a carga funcional"(3).

La oseointegración puede definirse como el contacto estable entre el hueso viable y remodelado con la superficie del implante, sin la interposición de tejido conectivo u otra cosa que no sea tejido óseo. Es en sí un contacto directo a nivel microscópico entre el hueso vivo y la superficie de un implante. La oseointegración es, por tanto, la conexión directa, estructural y funcional entre el hueso vivo bien organizado y la superficie del sustituto dental implantado que será capaz de absorber las fuerzas provenientes de las funciones propias del sistema estomatognático(4).

Para lograr esta oseointegración, es necesario realizar una preparación del lecho óseo de un diámetro tal que permita la colocación de un tornillo de titanio de manera ajustada a esta, con la finalidad de conseguir que este quede firmemente anclado al hueso. Sin embargo, algunas preparaciones quirúrgicas en el hueso alveolar pueden quedar con un diámetro mayor al del implante seleccionado en forma previa, el cual no obtiene la necesaria estabilidad primaria, lo que hace imposible la oseointegración deseada.

Algunas acciones que pueden llevar a una preparación de diámetro excesivo en el hueso alveolar son:

a) Hueso (clase III y/o IV) excesivamente blando (osteoporosis).

b) Exceso de fuerza, al roscar el implante.

c) Fresado muy repetido con el micromotor.

d) Error en la selección del diámetro del implante y la fresa.
Las soluciones más comunes para resolver estos problemas son: 1) Seleccionar un nuevo implante de mayor diámetro.

2) Colocar dentro de la preparación, hueso autólogo, heterólogo o hidroxiapatita pulverizados para obtener la fijación primaria del implante, y en consecuencia, su oseointegración ${ }^{(3)}$.

\section{MATERIALES Y MÉTODO}

Usando un disco de carburúndum, accionado por un motor de laboratorio, fueron cortadas cuñas de titanio desde una placa de titanio puro de $2 \mathrm{~mm}$ de espesor. El tamaño de estos segmentos fue de $3,5 \mathrm{x}$ $1,5 \mathrm{~mm}$ y de $5,5 \times 2,5 \mathrm{~mm}$ de largo/ancho (Figura 1). Se les limpió en una lavadora ultrasónica con agua destilada, luego, con alcohol de $70^{\circ}$ durante 30 minutos en cada baño en forma consecutiva; posterior a esto las cuñas de titanio fueron esterilizadas en autoclave, dentro de sobres individuales. Este sistema nos entrego cuñas separadas y selladas herméticamente (Figura 2).

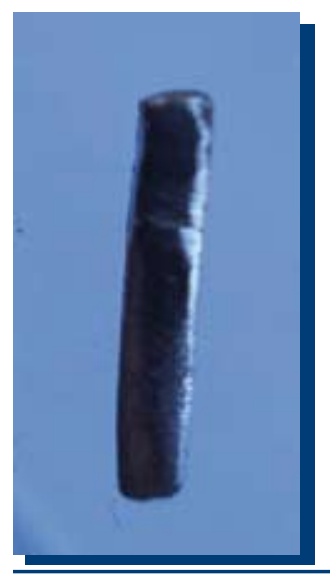

Figura 1. Cuña de titanio.

1. Universidad San Sebastián. Concepción, Chile.

Correspondencia autor: Christian Valenzuela. chvalenz@udec.cl. Trabajo recibido el 15/10/2009. Aprobado para su publicación el 02/12/2009. 


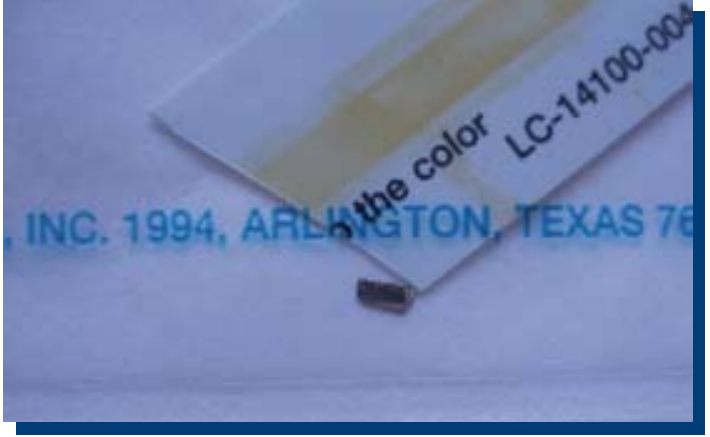

Figura 2. Cuña de titanio empacada

La elección del titanio para la cuña se debe a que este metal en contacto con la atmósfera se oxida en milisegundos transformándose su superficie en óxido de titanio. Dicho óxido se comporta como un material fisiológicamente bio-inerte, es decir que no produce rechazo, reacción natural del organismo ante la presencia de un cuerpo extraño que deriva habitualmente en complicaciones clínicas ${ }^{(5,6)}$.

Así, en aquellas situaciones en que en una cirugía un implante quedaba suelto en su lecho óseo, el paciente era informado en el momento acerca del problema y se le planteaba la posibilidad del uso de una cuña de titanio para fijar el implante original como tratamiento alternativo. Los pacientes que estuvieron de acuerdo con el procedimiento señalado, asentían en forma verbal dentro del pabellón y una vez finalizado el procedimiento, firmaron un consentimiento informado.

Se procedió entonces, cada vez, a extraer una cuña estéril de los sobres preparados con anterioridad, seleccionándola de acuerdo a la brecha observada entre el implante y la pared ósea, llevándola al sitio quirúrgico con un instrumento posicionador (como un porta agujas) e insertarla firmemente entre la pared del cilindro y el hueso, preferentemente en una cara proximal (Figura 3). En algunas ocasiones, se logró aumentar la fijación de los insertos de titanio al lecho, golpeando suavemente la cuña con la ayuda de un instrumento, para introducirla con buena presión entre el implante y la pared ósea proximal. Una vez insertada en su posición se comprobó que efectivamente se había conseguido la estabilidad primaria de ambos.

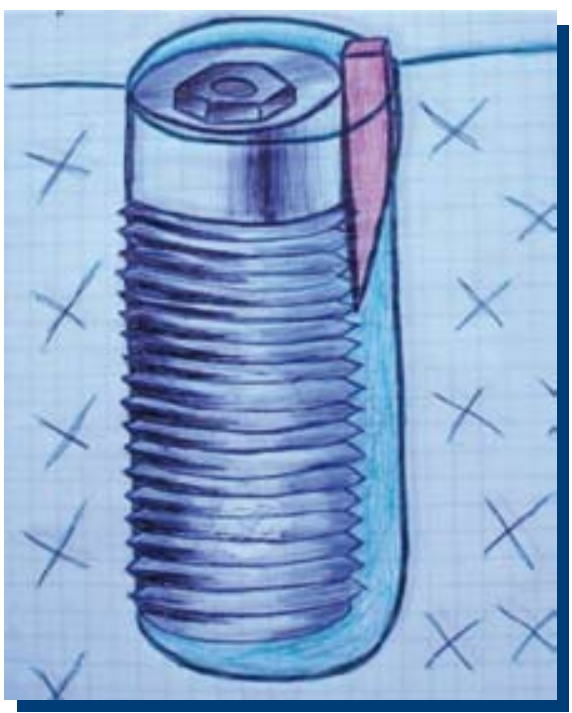

Figura 3. Dibujo esquemático de la cuña de titanio en su posición.

Luego de cada cirugía con instalación de cuñas se tomó radiografía de control como en este caso (Figura 4).

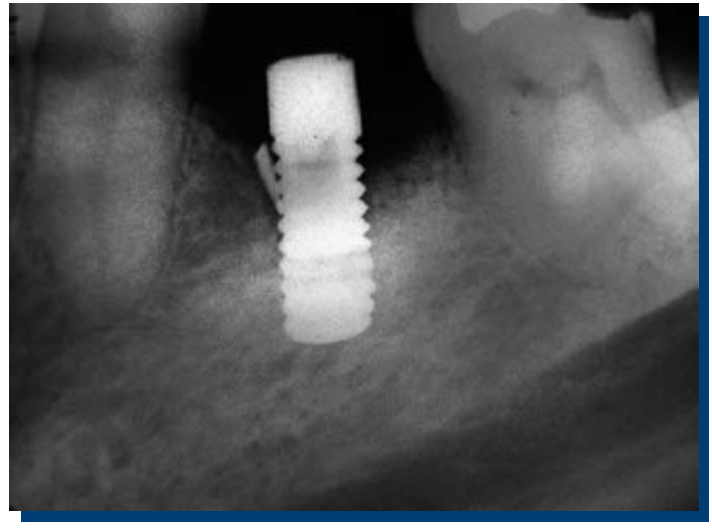

Figura 4. Radiografía sector pieza 3.6, se observa cuña de titanio implantada. Control a las 48 horas.

\section{RESULTADOS}

Se realizó el seguimiento de los casos tratados, tanto clínica como radiológicamente. Para evaluar la oseointegración se utilizaron los parámetros clínicos tradicionales de Albrektsson tal como fueron citados por Smith D. y Zarb G. . $^{(7)}$, junto a radiografías de control a los 6 meses (Figura 5), nos demostraron que los implantes se encontraban oseointegrados $^{(8)}$. Entre estos tenemos la ausencia de movilidad del implante en su lecho, junto con la ausencia de signos de inflamación, dolor o infección, ni alteraciones neurológicas de tipo sensitivo en los tejidos adyacentes; además de no presentar una interfase radiolúcida en las radiografías de control.

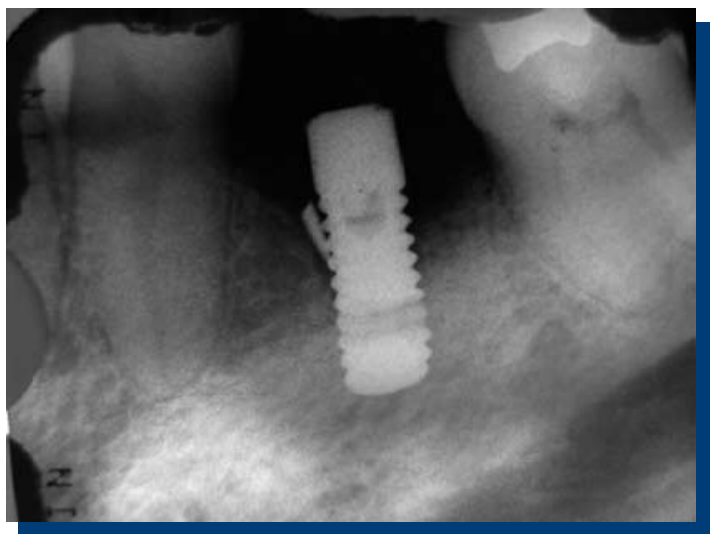

Figura 5. Radiografía sector pieza 3.6, se observa cuña de titanio implantada. Control a los 6 meses.

Con esta técnica se han solucionados alrededor de 4 casos, en algunos, en que la cuña quedó demasiado intruída en el alvéolo, se optó por dejarla en su lugar, no ocasionando problemas posteriores, al estar oseointegrada.

Después de 6 meses, en uno de los casos, tal como se aprecia en las radiografías y fotografías expuestas acá, se observó la cuña en su lugar, sin signos de enfermedad periodontal en relación a ella (Figura 6) y se procedió al retiro de esta con una pinza (Figura 7). 


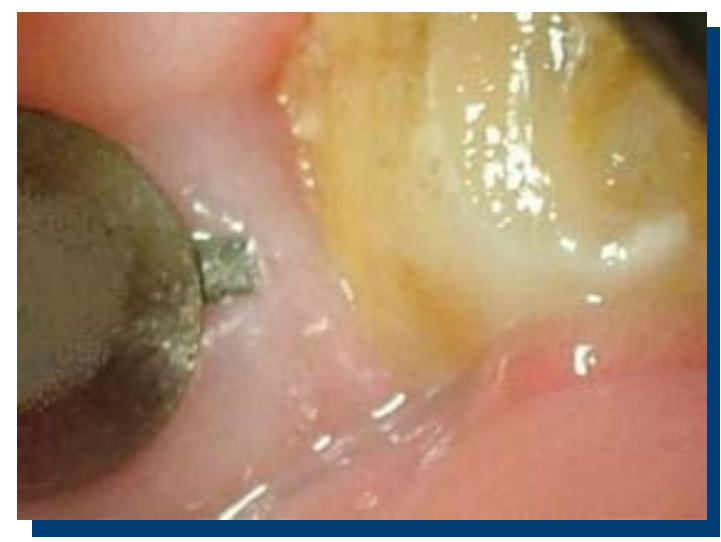

Figura 6. Control clínico a los 6 meses.

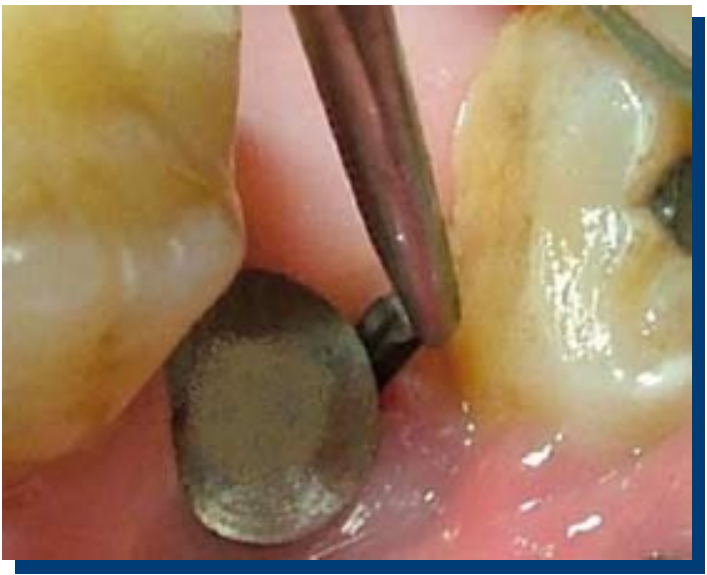

Figura 7. Retiro de cuña de titanio con pinzas.

Una vez retirada la cuña, en uno de los casos se pudo apreciar que evidenciaba signos de oseointegración, ya que presentaba tejido óseo adherido a ella, posiblemente debido a la superficie rugosa de ésta (Figuras 8 y 9 ).

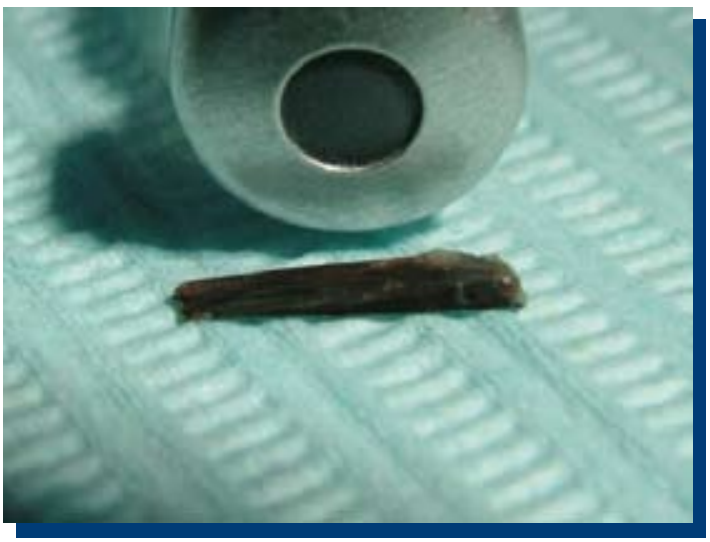

Figura 8. Comparación de tamaño de cuña con el diámetro de un tubo de anestesia. Obsérvese el resto óseo adherido a la superficie de está.

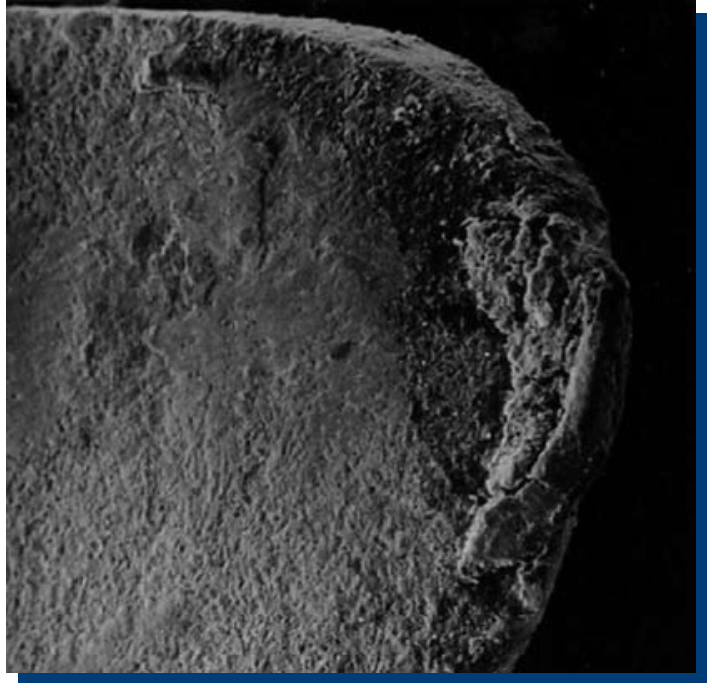

Figura 9. Fotografía S.E.M., borde rugoso de cuña de titanio.

Posteriormente al observarla al S.E.M. (microscopio electrónico de barrido) se pesquisó una célula compatible con osteocito (Figura 10).

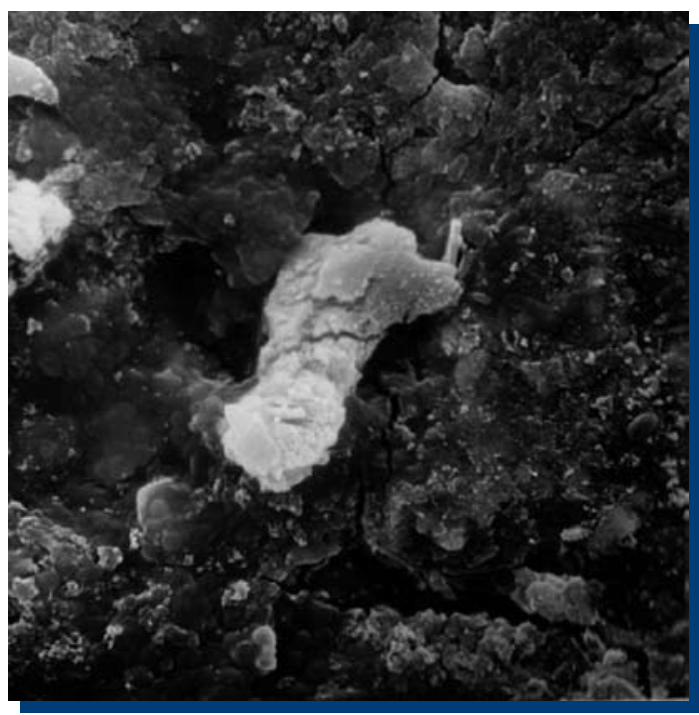

Figura 10. Fotografía S.E.M. Superficie de titanio con célula compatible con osteocito.

\section{DISCUSIÓN}

Durante mucho tiempo, la única forma de lograr la estabilidad primaria de los implantes en una preparación ósea de mayor diámetro ya sea por accidente o posterior a una exodoncia reciente (implantes inmediatos) $)^{(9)}$, ha sido la de sustituir el primer implante de titanio por otro de mayor diámetro, lo que en general implica un alto costo de mantenimiento de stock para el profesional.

En estos casos una posibilidad es la de colocar implantes de diámetro ancho, como los llamados implantes de rescate (Rescue Dental Internal Implant System, MegaGen Co., Ltd) o los implantes extra anchos (SM-Extra Wide (RBM), Implant System) $)^{(10)}$, los cuales pueden ser mantenidos en reserva para cualquier situación, durante la cirugía ósea, en el que el lecho por accidente exceda el diámetro de los implantes estandar.

El inconveniente, como ya fue mencionado, es el alto costo económico que esto implica. El uso de cuñas de titanio de diferente tamaño tiene la ventaja de ser una simple y económica forma para fijar los implantes que han quedado sueltos en el lecho óseo recién taladrado.

Esta técnica mantiene fijos los implantes hasta la formación de nuevo hueso alrededor de este. Luego de algún tiempo se logra una interfase de hueso neoformado entre el titanio y el hueso alveolar, 
que con el tiempo alcanzará la fuerza suficiente para permitir la función masticatoria. Posteriormente al retirar la cuña, el lecho dejado por ella es rellenado por nuevo hueso neoformado, el cual no es indispensable ya que el resto de la superficie del implante consiguió oseointegrarse.

Entre las desventajas que cabe mencionar, tenemos la posibilidad que la cuña se puede perder entre los tejidos blandos si el instrumento posicionador resbala, también la cuña podría producir una inclinación del implante dentro de su lecho quirúrgico; otro posible problema sería que se pueden producir fracturas de la tabla ósea opuesta, por exceso de fuerza al posicionar la cuña, por esto debemos tener la precaución de no afirmar la cuña en la fina tabla ósea vestibular de los maxilares, sino que debemos hacerlo en la zona proximal que es más sólida.

Por último, es necesaria investigación adicional para evaluar el rendimiento a largo plazo de las cuñas de titanio fijadas por este método, ya que no existen trabajos similares previos publicados en la literatura formal.

\section{AGRADECIMIENTOS}

Departamento de Microscopía Electrónica, Dirección de Investigación. Universidad de Concepción.

Doctor Guillermo Hardtmann Alarcón, fotografías clínicas.

\section{REFERENCIAS BIBLIOGRAFICAS}

1. Brånemark, P.I.; Breine, U.; Adell, R.; Hansson B.O.: Lindström, J. \& Ollson, A. Intra-osseous anchorage of dental prostheses. I. Experimental studies. Scandinavian Journal of Plastic \& Reconstructive Surgery 1969;3: 81-100.

2. García-Arocha, R.A.; García, V: Suárez, A. Rehabilitación protésica del maxilar inferior usando implantes óseointegrados de carga inmediata: Reporte de un caso. Acta odontol. venez. [online]. 2005, 43(2); 165170. Disponible en: <http://www.scielo.org.ve/scielo.php?script=sci_ arttext\&pid=S000163652005000200012\&Ing=es\&nrm=iso>

3. Lemus, L.M.; Justo, M.; Almagro, S.; Sáez, R.; Triana, K. Rehabilitación sobre implantes oseointegrados. A case with rehabilitation on boneintegrated implants. Revista Cubana de Estomatología. 2009;46(1). 4. Zeron y Gutiérrez, A. Oseointegración: serendipia o razonamiento científico. Revista Mexicana de Odontología Clínica. 2006; 1(4); 4-9.

5. Brånemark, P.I.; Zarb, G.A.; Albrektsson, T. Tissue-Integrated Prostheses: Osseointegration in Clinical Dentistry. Chicago: Quintessence, 1985; 99-111; 135-140.
6. Keller, J.C. Physical and Biological Characteristics of Implant Materials. Advances in Dental Research "Oral Biology and Dental Implants". 1999; 13, 5-7.

7. Smith D.; Zarb G. Criteria for success of osseointegrated endosseous implants. J Prosthet Dent. 1989; 62:567-572.

8. McKinney, R.V.; Steflick, D.E.; Koth, D.L.; Singh B.B. The Scientific Basis for Dental Implant Therapy. Journal of Dental Education. 1988; 52(12), 696-705.

9. Penarrocha, M.; Uribe, R.; Balaguer, J. Implantes inmediatos a la exodoncia: Situación actual. Med. oral patol. oral cir. bucal (Ed.impr.) [online]. 2004, vol.9, n.3 [citado 2009-11-27], pp. 234-235. Disponible en: $\quad$ http://scielo.isciii.es/scielo.php?script=sci_arttext\&pid=S1698$44472004000300009 \&$ Ing=es\&nrm=iso $>$. ISSN 1698-4447.

10. Requerimiento K080128 de Premarket Notification $(510(\mathrm{~K}))$ a la FDA por DIO Department, DSI, Inc. Disponible en:

http://www.accessdata.fda.gov/cdrh_docs/pdf8/K080128.pdf 